Research Article

\title{
Comparison Theorems for Single and Double Splittings of Matrices
}

\author{
Cui-Xia Li, Qun-Fa Cui, and Shi-Liang Wu \\ School of Mathematics and Statistics, Anyang Normal University, Anyang 455000, China \\ Correspondence should be addressed to Cui-Xia Li; lixiatk@126.com \\ Received 5 February 2013; Accepted 14 March 2013 \\ Academic Editor: Giuseppe Marino
}

Copyright ( 2013 Cui-Xia Li et al. This is an open access article distributed under the Creative Commons Attribution License, which permits unrestricted use, distribution, and reproduction in any medium, provided the original work is properly cited.

Some comparison theorems for the spectral radius of double splittings of different matrices under suitable conditions are presented, which are superior to the corresponding results in the recent paper by Miao and Zheng (2009). Some comparison theorems between the spectral radius of single and double splittings of matrices are established and are applied to the Jacobi and Gauss-Seidel double SOR method.

\section{Introduction}

Consider the linear system

$$
A x=b,
$$

where $A \in \mathbb{R}^{n \times n}$ is nonsingular, $b \in \mathbb{R}^{n \times 1}$ is given, and $x \in$ $\mathbb{R}^{n \times 1}$ is unknown. The splitting of the coefficient matrix

$$
A=M-N
$$

where $M$ is nonsingular, is called a single splitting of $A$ in [1]; the basic iterative method for solving (1) is

$$
x^{k+1}=M^{-1} N x^{k}+M^{-1} b \equiv T x^{k}+M^{-1} b, \quad k=0,1,2, \ldots,
$$

where matrix $T=M^{-1} N$ is the iteration matrix in (3). Obviously, the iterative method (3) converges to the unique solution of the linear system (1) if and only if the spectral radius $\rho\left(M^{-1} N\right)$ of the iteration matrix is smaller than 1 . The spectral radius of the iteration matrix is decisive for the convergence and stability, and the smaller it is, the faster the iterative method converges when the spectral radius is smaller than 1. So far, many comparison theorems of single splitting of matrices have been arisen in some papers and books [2-8].
The double splitting of $A$ was introduced by Woźnicki [1] and can be described as follows. Splitting the matrix $A$ in the form

$$
A=P-R-S
$$

is called the double splitting of $A$, where $P$ is a nonsingular matrix; the corresponding iterative scheme is spanned by three successive iterations:

$$
x^{k+1}=P^{-1} R x^{k}+P^{-1} S x^{k-1}+P^{-1} b, \quad k=0,1,2, \ldots
$$

Following the idea of Golub and Varga [9], Woźnicki wrote (5) in the following equivalent form:

$$
\left[\begin{array}{c}
x^{k+1} \\
x^{k}
\end{array}\right]=\left[\begin{array}{cc}
P^{-1} R & P^{-1} S \\
I & 0
\end{array}\right]\left[\begin{array}{c}
x^{k} \\
x^{k-1}
\end{array}\right]+\left[\begin{array}{c}
P^{-1} b \\
0
\end{array}\right],
$$

where $I$ is the identity matrix. Then, the iterative method (6) converges to the unique solution of (1) for all initial vectors $x^{0}, x^{1}$ if and only if the spectral radius of the iteration matrix

$$
W=\left[\begin{array}{cc}
P^{-1} R & P^{-1} S \\
I & 0
\end{array}\right]
$$

is less than one, that is, $\rho(W)<1$.

Recently, some comparison theorems for double splittings of monotone matrices and Hermitian positive definite matrices were presented in $[8,10-13]$. Elsner et al. [14] 
presented some comparison theorems of single splittings of different monotone matrices, that is, matrices with nonnegative inverses. Our basic purpose here is to derive some new comparison theorems for the spectral radius of double splittings of different matrices. Under suitable conditions, new comparison theorems are superior to the corresponding results in the recent paper [12]. Some comparison theorems between the spectral radius of single and double splittings of matrices are also established and are applied to the Jacobi and Gauss-Seidel double SOR method.

\section{Preliminaries}

For convenience, we give some of the notations, definitions, and lemmas which will be used in the sequel.

The matrix $A$ is called nonnegative and denoted by $A \geq 0$ if $a_{i j} \geq 0$ for $i, j=1,2, \ldots, n$. We write $A \geq B(A>B)$ if $a_{i j} \geq b_{i j}\left(a_{i j}>b_{i j}\right)$ for $i, j=1,2, \ldots, n$. The matrix $A$ is called a monotone matrix if $A^{-1} \geq 0$. Matrix $A$ is an $L$-matrix if $a_{i i}>0 \quad(i=1, \ldots, n)$ and $a_{i j}<0$ for all $i, j=1, \ldots, n ; i \neq j$.

Definition 1. Let $A$ be a nonsingular matrix. Then, the double splitting $A=P-R-S$ is

(i) convergent if and only if $\rho(W)<1$;

(ii) a regular double splitting if $P^{-1} \geq 0, R \geq 0$ and $S \geq 0$;

(iii) a weak regular double splitting if $P^{-1} \geq 0, P^{-1} R \geq 0$, and $P^{-1} S \geq 0$;

(iv) a nonnegative splitting if $P^{-1} R \geq 0$ and $P^{-1} S \geq 0$.

Lemma 2 (see [3]). Let $A \geq 0$. Then, $\alpha x \leq A x, x \geq 0$, implies $\alpha \leq \rho(A)$ and $A x \leq \beta x, x>0$, implies $\rho(A) \leq \beta$.

Lemma 3 (see [10]). Let $A^{-1} \geq 0$ and $A=P-R-S$ be a weak regular double splitting. Then, $\rho(W)<1$.

\section{Comparison Theorem}

In [12], Miao and Zheng gave a comparison theorem for the spectral radius of double splittings of different monotone matrices. That is, [12, Theorem 3.1] is a major result and is described as follows.

Let $A_{1}$ and $A_{2}$ be two monotone matrices, and let $A_{1}=$ $P_{1}-R_{1}-S_{1}$, and let $A_{2}=P_{2}-R_{2}-S_{2}$ be double splittings of $A_{1}$ and $A_{2}$, respectively. Consequently,

$$
W_{1}=\left[\begin{array}{cc}
P_{1}^{-1} R_{1} & P_{1}^{-1} S_{1} \\
I & 0
\end{array}\right], \quad W_{2}=\left[\begin{array}{cc}
P_{2}^{-1} R_{2} & P_{2}^{-1} S_{2} \\
I & 0
\end{array}\right] \text {. }
$$

Theorem 4 (see [12]). Let $A_{1}$ and $A_{2}$ be two nonsingular matrices with $A_{1}^{-1} \geq 0$ and $A_{2}^{-1} \geq 0, A_{1}=P_{1}-R_{1}-S_{1}$, and let $A_{2}=P_{2}-R_{2}-S_{2}$ be weak regular double splittings. If $P_{1}^{-1} A_{1} \geq P_{2}^{-1} A_{2}$ and $P_{1}^{-1} R_{1} \geq P_{2}^{-1} R_{2}$, then $\rho\left(W_{1}\right) \leq \rho\left(W_{2}\right)<$ 1 .

Based on the forms of $W_{1}$ and $W_{2}$, we have the following theorem.
Theorem 5. Let $A_{1}$ and $A_{2}$ be two nonsingular matrices, and let $A_{1}=P_{1}-R_{1}-S_{1}$ and $A_{2}=P_{2}-R_{2}-S_{2}$ be nonnegative splittings. If $P_{1}^{-1} R_{1} \leq P_{2}^{-1} R_{2}$ and $P_{1}^{-1} S_{1} \leq P_{2}^{-1} S_{2}$, then $\rho\left(W_{1}\right) \leq$ $\rho\left(W_{2}\right)<1$ for $\rho\left(W_{2}\right)<1$.

Proof. Obviously, if $P_{1}^{-1} R_{1} \leq P_{2}^{-1} R_{2}$ and $P_{1}^{-1} S_{1} \leq P_{2}^{-1} S_{2}$, then $0 \leq W_{1} \leq W_{2}$. Therefore, we obtain that $\rho\left(W_{1}\right) \leq \rho\left(W_{2}\right)<1$ for $\rho\left(W_{2}\right)<1$.

Based on Definition 1, we obtain the following Theorem 6, which is superior to Theorem 4 [12].

Theorem 6. Let $A_{1}$ and $A_{2}$ be two nonsingular matrices, and let $A_{1}=P_{1}-R_{1}-S_{1}$ and $A_{2}=P_{2}-R_{2}-S_{2}$ be nonnegative splittings. If $P_{1}^{-1} A_{1} \geq P_{2}^{-1} A_{2}$ and $P_{1}^{-1} R_{1} \geq P_{2}^{-1} R_{2}$, then $\rho\left(W_{1}\right) \leq \rho\left(W_{2}\right)<1$ for $0<\rho\left(W_{2}\right)<1$.

Proof. Obviously, $W_{1} \geq 0$ and $W_{2} \geq 0$. By the PerronFrobenius theorem [3], there exists a vector

$$
x=\left[\begin{array}{l}
x_{1} \\
x_{2}
\end{array}\right] \geq 0, \quad x \neq 0
$$

such that $W_{2} x=\rho\left(W_{2}\right) x$; that is,

$$
\begin{gathered}
P_{2}^{-1} R_{2} x_{1}+P_{2}^{-1} S_{2} x_{2}=\rho\left(W_{2}\right) x_{1}, \\
x_{1}=\rho\left(W_{2}\right) x_{2} .
\end{gathered}
$$

Then, we have

$$
\begin{aligned}
& W_{1} x-\rho\left(W_{2}\right) x \\
& =\left[\begin{array}{c}
P_{1}^{-1} R_{1} x_{1}+P_{1}^{-1} S_{1} x_{2}-\rho\left(W_{2}\right) x_{1} \\
x_{1}-\rho\left(W_{2}\right) x_{2}
\end{array}\right] \\
& =\left[\begin{array}{c}
\left(P_{1}^{-1} R_{1}-P_{2}^{-1} R_{2}\right) x_{1}+\frac{1}{\rho\left(W_{2}\right)}\left(P_{1}^{-1} S_{1}-P_{2}^{-1} S_{2}\right) x_{1} \\
x_{1}-\rho\left(W_{1}\right) x_{2}
\end{array}\right] \\
& \leq \frac{1}{\rho\left(W_{2}\right)}\left[\begin{array}{c}
\left(P_{1}^{-1} R_{1}-P_{2}^{-1} R_{2}\right) x_{1}+\left(P_{1}^{-1} S_{1}-P_{2}^{-1} S_{2}\right) x_{1} \\
0
\end{array}\right] \\
& =\frac{1}{\rho\left(W_{2}\right)}\left[\begin{array}{c}
\left(P_{1}^{-1} R_{1}+P_{1}^{-1} S_{1}\right) x_{1}-\left(P_{2}^{-1} R_{2}+P_{2}^{-1} S_{2}\right) x_{1} \\
0
\end{array}\right] \\
& =\frac{1}{\rho\left(W_{2}\right)}\left[\begin{array}{c}
P_{1}^{-1}\left(R_{1}+S_{1}\right) x_{1}-P_{2}^{-1}\left(R_{2}+S_{2}\right) x_{1} \\
0
\end{array}\right] \\
& =\frac{1}{\rho\left(W_{2}\right)}\left[\begin{array}{c}
P_{1}^{-1}\left(P_{1}-A_{1}\right) x_{1}-P_{2}^{-1}\left(P_{2}-A_{2}\right) x_{1} \\
0
\end{array}\right] \\
& =\frac{1}{\rho\left(W_{2}\right)}\left[\begin{array}{c}
\left(P_{2}^{-1} A_{2}-P_{1}^{-1} A_{1}\right) x_{1} \\
0
\end{array}\right] \\
& \leq 0 \text {. }
\end{aligned}
$$

From Lemma 2, we obtain that $\rho\left(W_{1}\right) \leq \rho\left(W_{2}\right)<1$ for $0<$ $\rho\left(W_{2}\right)<1$. 
By investigating Theorem 6, it is easy to see that the conditioners, Theorem 6 are weaker than those of Theorem 4 [12]. That is, the result of Theorem 6 holds without $A_{1}^{-1} \geq 0$ and $A_{2}^{-1} \geq 0$.

Similarly, we have the following result.

Theorem 7. Let $A_{1}$ and $A_{2}$ be two nonsingular matrices, and let $A_{1}=P_{1}-R_{1}-S_{1}$ and $A_{2}=P_{2}-R_{2}-S_{2}$ be nonnegative splittings. If $P_{1}^{-1} A_{1} \geq P_{2}^{-1} A_{2}$ and $P_{1}^{-1} S_{1} \leq P_{2}^{-1} S_{2}$, then $\rho\left(W_{1}\right) \leq$ $\rho\left(W_{2}\right)<1$ for $0<\rho\left(W_{2}\right)<1$.

\section{Convergence for the Jacobi and Gauss-Seidel Double SOR Method}

To establish some comparison theorems between the spectral radius of single and double splittings of matrices, based on (3) and (5), we obtain that $M=P$ and $N=R+S$. Here and now, $T=P^{-1}(R+S)$.

The result for comparing $\rho(W)$ with $\rho(T)$ is stated as in the following theorem.

Theorem 8. Let $A=P-R-S$ be a nonnegative splitting. Then,

(1) $\rho(T) \leq \rho(W)<1$ for $\rho(W)<1$;

(2) $\rho(T) \geq \rho(W)>1$ for $\rho(W)>1$.

Proof. By Definition 1, obviously, matrix $W \geq 0$. Based on the Perron-Frobenius theorem [3], there exists a vector

$$
x=\left[\begin{array}{l}
x_{1} \\
x_{2}
\end{array}\right] \geq 0, \quad x \neq 0
$$

such that $W x=\rho(W) x$, that is,

$$
\left[\begin{array}{cc}
P^{-1} R & P^{-1} S \\
I & 0
\end{array}\right]\left[\begin{array}{l}
x_{1} \\
x_{2}
\end{array}\right]=\rho(W)\left[\begin{array}{l}
x_{1} \\
x_{2}
\end{array}\right]
$$

The above equation is equivalent to

$$
\begin{gathered}
P^{-1} R x_{1}+P^{-1} S x_{2}=\rho(W) x_{1}, \\
x_{1}=\rho(W) x_{2} .
\end{gathered}
$$

From (15), we get that $x_{2}=(1 / \rho(W)) x_{1}$. Substituting it into (14) yields

$$
\rho(W) x_{1}=P^{-1} R x_{1}+\frac{1}{\rho(W)} P^{-1} S x_{1} \text {. }
$$

If $\rho(W)<1$, then

$$
\rho(W) x_{1} \geq P^{-1} R x_{1}+P^{-1} S x_{1} \text {. }
$$

That is, $\rho(W) x_{1} \geq T x_{1}$. By Lemma 2 , it is easy to obtain that $\rho(T) \leq \rho(W)<1$.

Obviously, we also obtain that $\rho(T) \geq \rho(W)>1$ for $\rho(W)>1$.
Example 9. Let

$$
\begin{aligned}
A & =\left[\begin{array}{cc}
1 & -2 \\
-2 & 1
\end{array}\right] \\
& =\left[\begin{array}{cc}
0 & -2 \\
-2 & 0
\end{array}\right]-\left[\begin{array}{cc}
-1 & 0 \\
0 & 0
\end{array}\right]-\left[\begin{array}{cc}
0 & 0 \\
0 & -1
\end{array}\right] \\
& \equiv P-R-S .
\end{aligned}
$$

Then,

$$
\begin{aligned}
& P^{-1}=\left[\begin{array}{cc}
0 & -\frac{1}{2} \\
-\frac{1}{2} & 0
\end{array}\right] \leq 0, \\
& P^{-1} R=\left[\begin{array}{ll}
0 & 0 \\
\frac{1}{2} & 0
\end{array}\right] \geq 0, \\
& P^{-1} S=\left[\begin{array}{ll}
0 & \frac{1}{2} \\
0 & 0
\end{array}\right] \geq 0 .
\end{aligned}
$$

By the simple computations, we have $\rho(W)=0.63$ and $\rho(T)=$ 0.5 . Clearly, $\rho(T) \leq \rho(W)<1$ holds.

Let the matrix $A$ be split as

$$
A=D-L-U
$$

where $D=\operatorname{diag}(A)$, and $L, U$ are strictly lower and upper triangular matrices, respectively, for $\omega \neq 0$ and $\sigma \neq 0$. Let

$$
\begin{aligned}
& P_{J}=\frac{1}{\omega \sigma} D, \\
& R_{J}=\frac{1}{\omega \sigma}[\omega \sigma(L+U)-(\omega-1) D-(\sigma-1) D], \\
& S_{J}=\frac{(\omega-1)(\sigma-1)}{\omega \sigma} D .
\end{aligned}
$$

Then, the iterative method (5) corresponding to the double splitting

$$
A=P_{J}-R_{J}-S_{J}
$$

is called the Jacobi double SOR method $[1,15]$.

Based on (21), we have the following lemma.

Lemma 10. Let $A$ be an L-matrix, and let the double splittings be defined by (21) and (22). Suppose

$$
2-\sigma-\omega \geq 0, \quad(\omega-1)(\sigma-1) \geq 0 .
$$

Then, the double splitting defined by (22) is regular.

$$
W_{J}=\left[\begin{array}{cc}
P_{J}^{-1} R_{J} & P_{J}^{-1} S_{J} \\
I & 0
\end{array}\right], \quad T_{J}=P_{J}^{-1}\left(R_{J}+S_{J}\right) .
$$

Then, we have the following result. 
Theorem 11. Under the conditions of Lemma 10, then

$$
\begin{aligned}
& \text { (1) } \rho\left(T_{J}\right) \leq \rho\left(W_{J}\right)<1 \text { for } \rho\left(W_{J}\right)<1 \text {; } \\
& \text { (2) } \rho\left(T_{J}\right) \geq \rho\left(W_{J}\right)>1 \text { for } \rho\left(W_{J}\right)>1 \text {. }
\end{aligned}
$$

Proof. From Theorem 8, it is easy to see that Theorem 11 holds.

$$
\begin{aligned}
& \text { Let } \\
& \begin{array}{l}
P_{G}=\frac{1}{\omega \sigma} D\left(I-\sigma D^{-1} U\right), \\
S_{G}=\frac{(\omega-1)(\sigma-1)}{\omega \sigma} D, \\
R_{G}=\frac{1}{\omega \sigma}\left[\omega \sigma L-(\omega-1) D\left(I-\sigma D^{-1} U\right)-(\sigma-1) D\right] .
\end{array}
\end{aligned}
$$

Then, the iterative method (5) corresponding to the double splitting

$$
A=P_{G}-R_{G}-S_{G}
$$

is called the Gauss-Seidel double SOR method $[1,15]$.

Let

$$
W_{G}=\left[\begin{array}{cc}
P_{G}^{-1} R_{G} & P_{G}^{-1} S_{G} \\
I & 0
\end{array}\right], \quad T_{G}=P_{G}^{-1}\left(R_{G}+S_{G}\right) .
$$

Similarly, we have the following result.

Theorem 12. Let $A$ be an L-matrix, and let the double splittings be defined by (25) and (26), for $0<\sigma \leq 1$ and $0<\omega \leq 1$; then

(1) $\rho\left(T_{G}\right) \leq \rho\left(W_{G}\right)<1$ for $\rho\left(W_{G}\right)<1$;

(2) $\rho\left(T_{G}\right) \geq \rho\left(W_{G}\right)>1$ for $\rho\left(W_{G}\right)>1$.

From Theorems 8, 11, and 12, it is easy to see that the spectral radius of single splitting method is less than the spectral radius of double splitting method under suitable conditions. That is, the efficiency of the single splitting method maybe be superior to that of the double splitting method under suitable conditions.

\section{Acknowledgment}

This research was supported by NSFC Tianyuan Mathematics Youth Fund (11026040, 11226337), Science \& Technology Development Plan of Henan Province (no. 122300410316) and by Natural Science Foundations of Henan Province (no. 13A110022).

\section{References}

[1] Z. I. Woźnicki, "Estimation of the optimum relaxation factors in partial factorization iterative methods," SIAM Journal on Matrix Analysis and Applications, vol. 14, no. 1, pp. 59-73, 1993.

[2] R. S. Varga, Matrix Iterative Analysis, vol. 27 of Springer Series in Computational Mathematics, Springer, Berlin, Germany, 2000.
[3] A. Berman and R. J. Plemons, Nonnegative Matrices in the Mathematics Sciences, SIAM, Philadelphia, Pa, USA, 1994.

[4] D. M. Young, Iterative Solution of Large Linear Systems, Academic Press, New York, NY, USA, 1971.

[5] Z. I. Woźnicki, "Nonnegative splitting theory," Japan Journal of Industrial and Applied Mathematics, vol. 11, no. 2, pp. 289-342, 1994.

[6] Z. I. Woźnicki, "Basic comparison theorems for weak and weaker matrix splittings," Electronic Journal of Linear Algebra, vol. 8, pp. 53-59, 2001.

[7] M. Benzi and D. B. Szyld, "Existence and uniqueness of splittings for stationary iterative methods with applications to alternating methods," Numerische Mathematik, vol. 76, no. 3, pp. 309-321, 1997.

[8] J. Song and Y. Song, "Convergence for nonnegative double splittings of matrices," Calcolo, vol. 48, no. 3, pp. 245-260, 2011.

[9] G. H. Golub and R. S. Varga, "Chebyshev semi-iterative methods, successive over-relaxation iterative methods, and second order Richardson iterative methods," Numerische Mathematik, vol. 3, pp. 147-156, 1961.

[10] S. Q. Shen and T. Z. Huang, "Convergence and comparison theorems for double splittings of matrices," Computers \& Mathematics with Applications, vol. 51, no. 12, pp. 1751-1760, 2006.

[11] S. Q. Shen, T. Z. Huang, and J. L. Shao, "Convergence and comparison results for double splittings of Hermitian positive definite matrices," Calcolo, vol. 44, no. 3, pp. 127-135, 2007.

[12] S. X. Miao and B. Zheng, "A note on double splittings of different monotone matrices," Calcolo, vol. 46, no. 4, pp. 261-266, 2009.

[13] C. Y. Zhang, "On convergence of double splitting methods for non-Hermitian positive semidefinite linear systems," Calcolo, vol. 47, no. 2, pp. 103-112, 2010.

[14] L. Elsner, A. Frommer, R. Nabben, H. Schneider, and D. B. Szyld, "Conditions for strict inequality in comparisons of spectral radii of splittings of different matrices," Linear Algebra and its Applications, vol. 363, pp. 65-80, 2003.

[15] L. J. Cvetković, “Two-sweep iterative methods," Nonlinear Analysis: Theory, Methods \& Applications, vol. 30, no. 1, pp. 2530, 1997. 


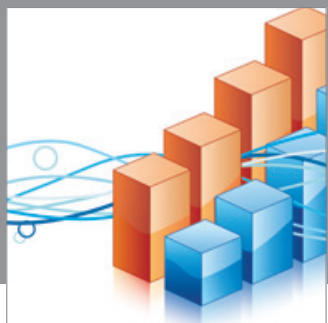

Advances in

Operations Research

mansans

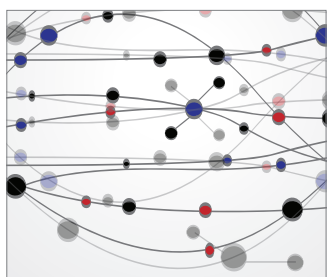

The Scientific World Journal
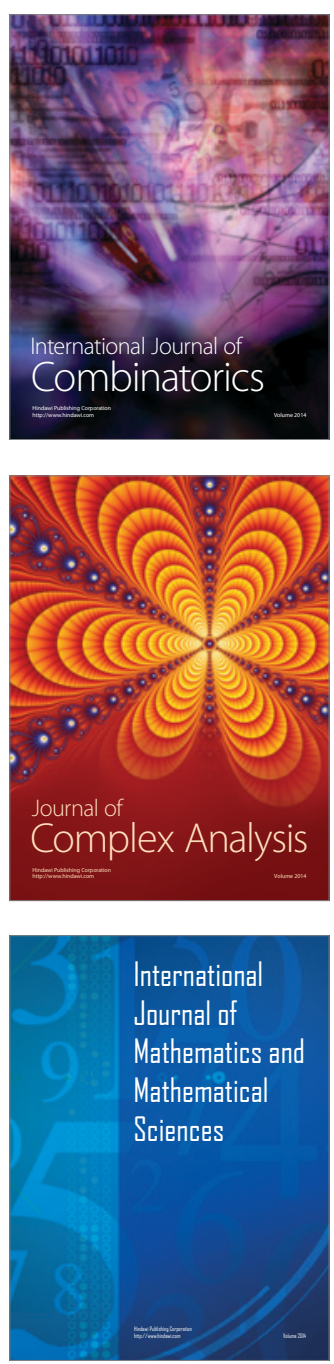
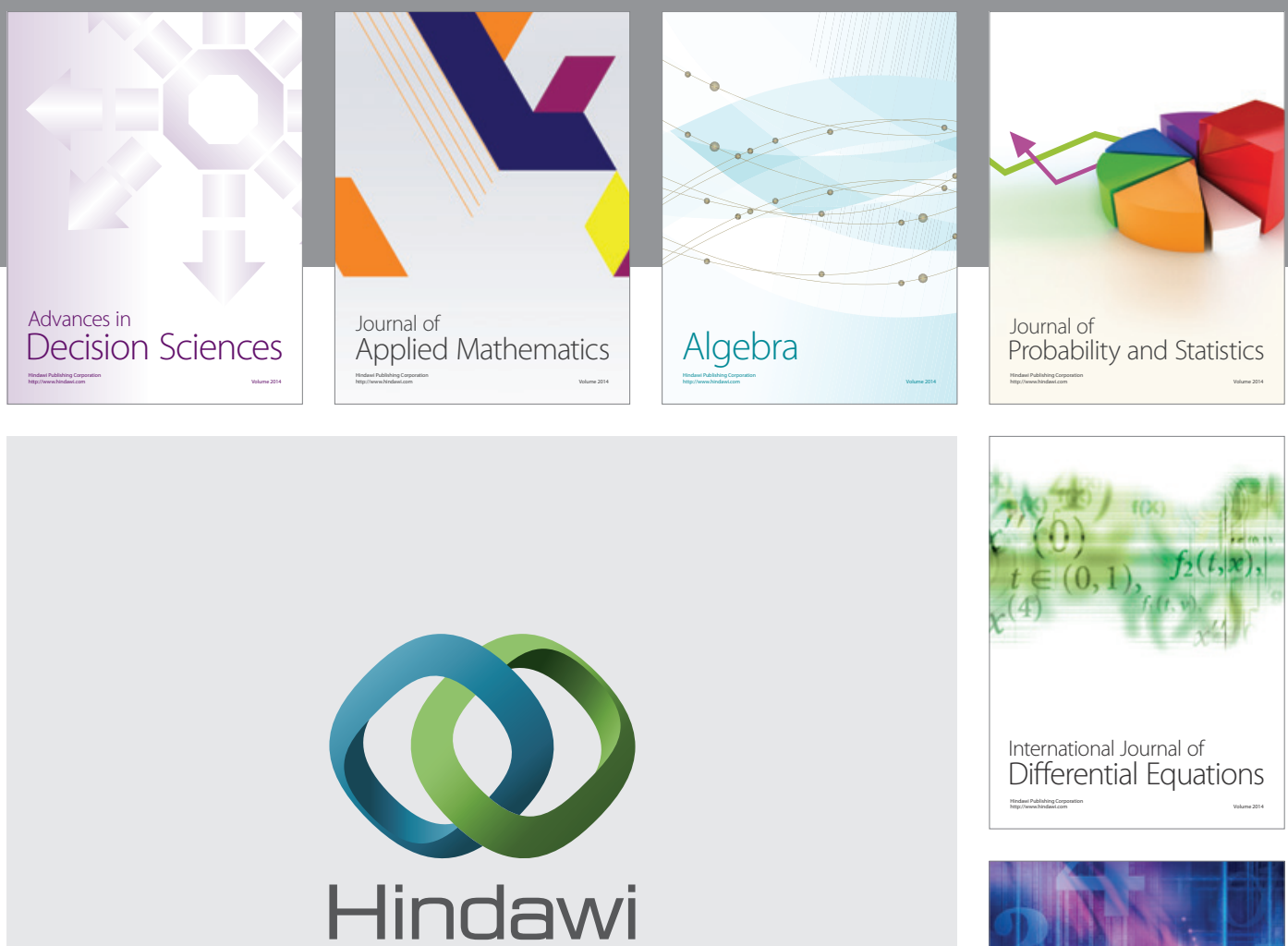

Submit your manuscripts at http://www.hindawi.com
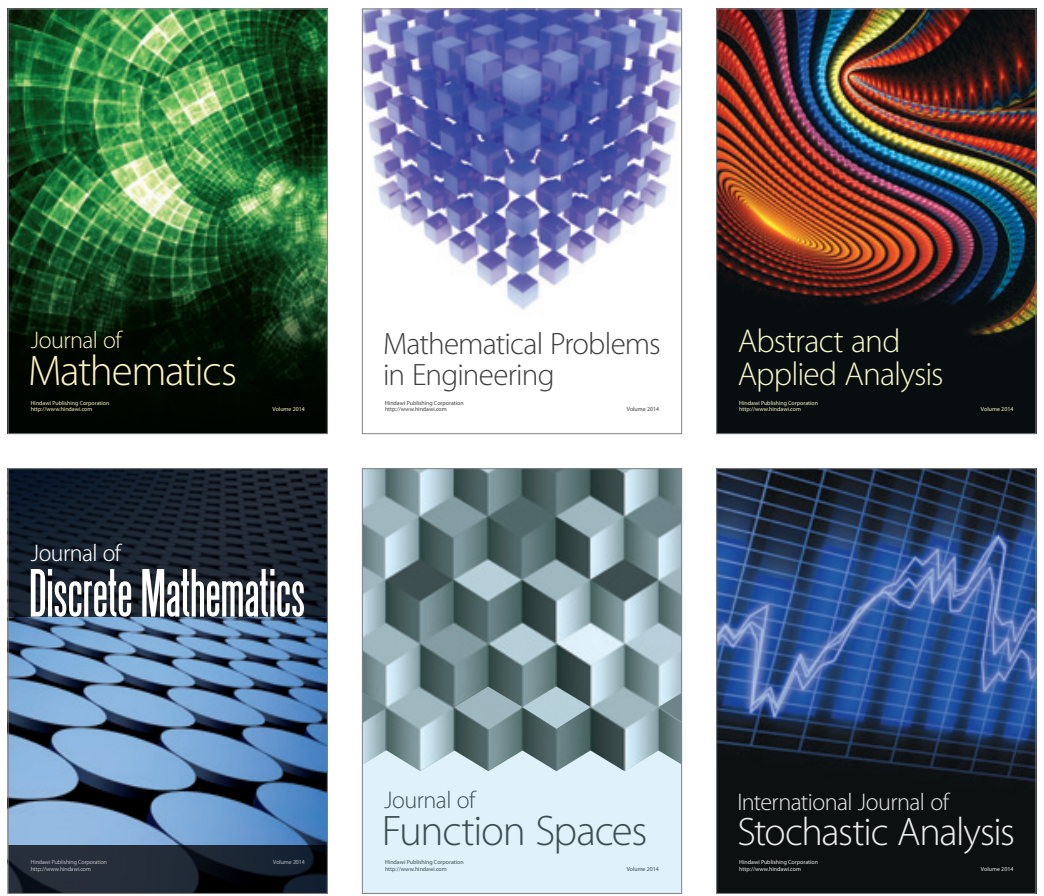

Journal of

Function Spaces

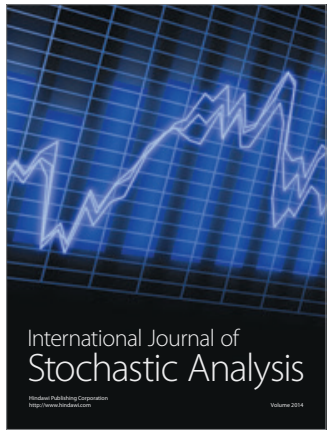

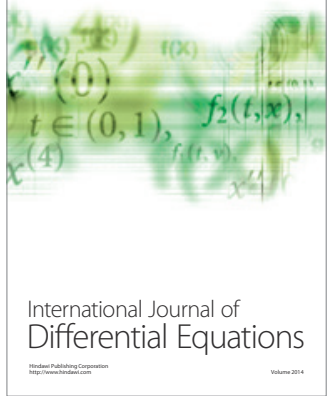
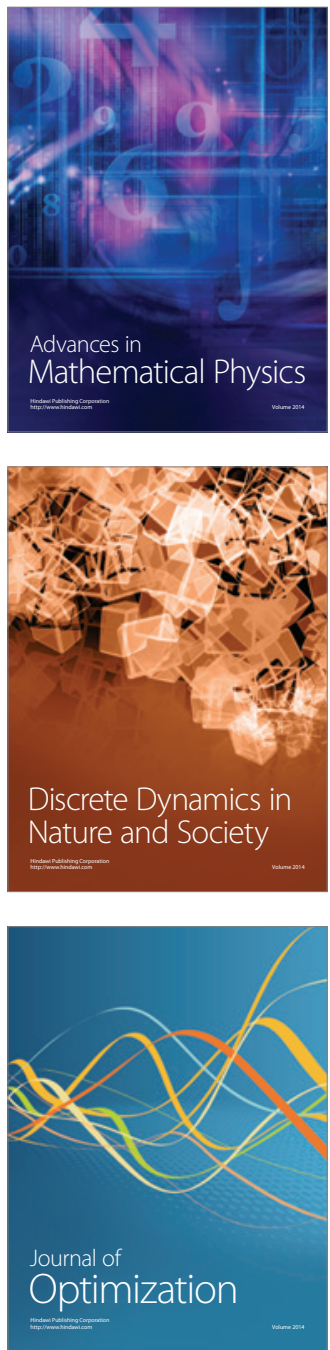